\title{
Oscillation flow induced by underwater supersonic gas jets
}

\author{
Hong-Hui Shi · Qiang Guo • Chao Wang • \\ Ruo-Ling Dong · Li-Te Zhang · Hui-Xia Jia • \\ Xiao-Gang Wang $\cdot$ Bo-Yi Wang
}

Received: 29 June 2009 / Revised: 20 April 2010 / Accepted: 8 July 2010 / Published online: 25 July 2010

(C) Springer-Verlag 2010

\begin{abstract}
This paper describes an experimental study on the oscillation flow characteristics of submerged supersonic gas jets issued from Laval nozzles. The flow pattern during the jet development and the jet expansion feedback phenomenon are studied using a high-speed camera and a pressure measurement system. The experimental results indicate that along the downstream distance, the jet has three flow regimes: (1) momentum jet; (2) buoyant jet; (3) plume. In the region near the nozzle exit a so-called bulge phenomenon is found. Bulging of the jet occurs many times before the more violent jet expansion feedback occurs. During the feedback process, the jet diameter can become several times that of the original one depending on the jet Mach number. The frequencies of the jet bulging and the jet expansion feedback are measured.
\end{abstract}

Keywords Underwater supersonic gas jet - Jet expansion feedback · Jet bulge $\cdot$ High-speed photography

Communicated by H. Kleine.

This paper was based on work that was presented at the 27th International Symposium on Shock Waves, St. Petersburg, Russia, July 19-24, 2009.

H.-H. Shi $(\varangle)$ · Q. Guo · C. Wang · R.-L. Dong · L.-T. Zhang ·

H.-X. Jia $\cdot$ X.-G. Wang

College of Mechanical Engineering and Automation,

Zhejiang Sci-Tech University, Hangzhou 310018, Zhejiang, China

e-mail: hhshi@zstu.edu.cn

B.-Y. Wang

State Key Laboratory of Nonlinear Mechanics, Institute of Mechanics,

Chinese Academy of Sciences, Beijing 100190, China

\section{Introduction}

Investigation of the hydrodynamic behavior of submerged gas jets and their effects is important in a wide range of natural and engineering processes such as volcano eruption in deep sea [1], water destratification in reservoirs, aeration treatment of wastewater, underwater cutting, pneumatic steelmaking, underwater jet-propulsion vehicles etc. A better understanding of the relevant phenomena and mechanisms in the submerged gas jets systems is necessary for optimizing various industrial processes. A review paper on this subject has been recently given by Gulawani et al. [2].

The research interest of underwater propulsion by gas jet has lasted for about half a century. In 1969, Ferguson [3] reported some results of flow separation of underwater nitrogen gas exhausting from Laval nozzles. In 2002, Todman and Wallis [4] tested the thrust of a steam jet propulsion unit. In 2006, Linck et al. [5] conducted experiments of submerged reacting and non-reacting flows from a combustion chamber into a water tank. Using un-choked and choked air jets at a maximum chamber pressure of $0.2 \mathrm{MPa}$, the Strouhal number associated with the cycle in the two-phase flow case was found to be two orders of magnitude smaller than that associated with large-scale jet mixing instabilities in single-phase mixing [5]. In 2007, Weiland et al. [6] reported an experimental study of underwater annular supersonic gas jet injection into a cross-flow. In 2009, Linck et al. [7] discussed that the instability of an underwater gas jet may be related to Richtmyer-Meshkov instability because the acoustic waves would have push the gas phase into the liquid phase.

The fluid mechanics study actually began in the area of metallurgy when tuyere refractory erosion was found (tuyere is the French term for a pipe or nozzle through which air is forced into a blast furnace, forge, etc.). With a relatively 
clear view of the fluid dynamic approach, Hoefele and Brimacombe [8] carried out experimental research on characterizing the flow regime of underwater gas jets from a submerged tuyere. Ozawa et al. [9] and Ozawa and Mori [10] performed an experiment of nitrogen gas injection into a mercury pool and found that the jet experiences bubbling-to-jetting transition when the jet velocity becomes sonic. Aoki et al. [11] and Aoki [12] first identified the "back-attack" phenomenon of an underwater sonic gas jet, that is, the jet periodically blows back to impact on the tuyere surface. Through the measurement of the impact force on the tuyere surface, "back-attack" frequencies of $5.0-6.7 \mathrm{~Hz}$ were found [11,12].

As indicated by Shi et al. [13], the current understanding of underwater supersonic gas jets is far from complete. Our study on this subject began in 2000. The static pressure measurement along the downstream of a sonic air jet by Qi et al. [14] indicates that there exists a shock-cell structure at the nozzle exit, which is in agreement with results of Loth and Faeth [15]. In the recent heat transfer experiment of a submerged condensing supersonic steam jet, Shao et al. [16] also suggest that the shock waves at the nozzle exit may cause the jet to expand radially. Furthermore, Dai et al. [17] and Wang et al. [18] have found that for underwater supersonic gas jets, the so-called "back-attack" phenomenon always exists no matter whether the jet is under-, fully- or over-expanded. This stimulates the interest of investigating the physics of the phenomenon. It is known that disturbances cannot propagate upstream in a supersonic flow. However, when the gas is injected into a liquid, a shear layer at the gas/liquid boundary forms and it can be subsonic. For a supersonic gas jet in open air, the screech tone principle has been established $[19,20]$, that is, wave feedback appears from the shear layer. Therefore, Shi et al. [21] propose that the "back-attack" phenomenon may be better explained as shock wave feedback phenomenon. In this paper, we describe the "backattack" as a jet expansion feedback phenomenon to distinguish it from the jet oscillations which have smaller amplitude. Wang et al. [22] have found that before the appearance of a jet expansion feedback (with a frequency $f_{w}=$ $5.3 \mathrm{~Hz}$ ), the jet bulging appears several times in advance and its frequency $f_{b}$ is greater than $f_{w}$. In this paper, we will provide more experimental results of the wave feedback and the bulge of the jet.

Figure 1 shows a conceptual sketch of an underwater supersonic jet. A shock-cell structure and a gas/liquid boundary co-exist in the flow field. Obviously, this kind of flow field usually cannot be stable. In the figure, Ma means the design Mach number and Me means the jet Mach number behind the incident inclined shock wave. Figure 2 shows a typical result of a pressure measurement of an underwater supersonic air jet in a three-dimensional water tank $(280 \mathrm{~cm}$ long, $50 \mathrm{~cm}$ wide and $55 \mathrm{~cm}$ high) $[17,18]$. The pressure signals are

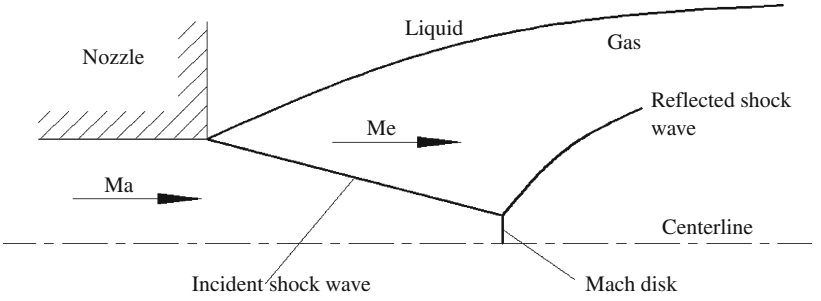

Fig. 1 Conceptual sketch of an underwater supersonic gas jet. Ma is the design Mach number. Me is the jet Mach number behind the incident inclined shock wave

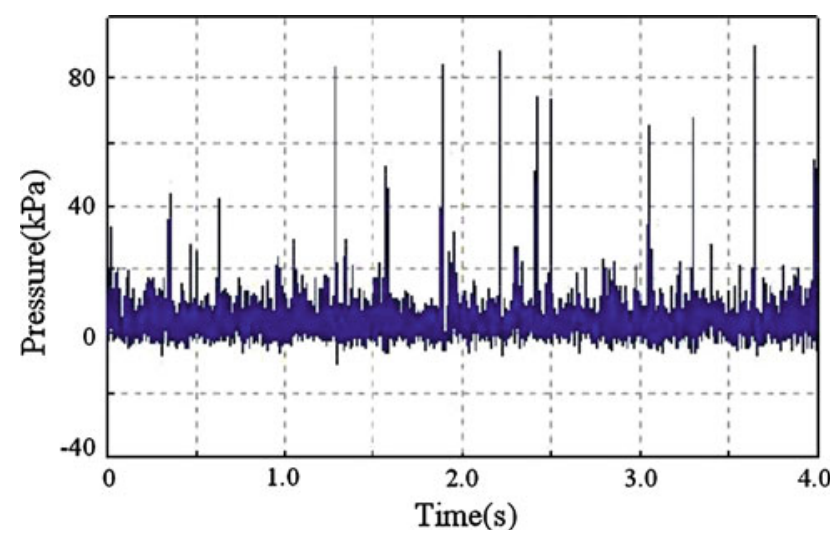

Fig. 2 Pressure measurement of the shock wave feedback of underwater under-expanded air jet at the nozzle exit centerline. The nozzle throat and exit diameters are 4.3 and $4.7 \mathrm{~mm}$, respectively. The jet Mach number at full-expansion condition (design Mach number) is $\mathrm{Ma}=1.5$. The water depth is $H=15 \mathrm{~cm}$. Measurement position: $10 \mathrm{~mm}$ from the nozzle exit. From Ref. [18]. The operation conditions can be seen in Ref. [21]

composed of (1) high amplitude and low frequency pressures caused by the "back-attack" or the jet expansion feedback; (2) medium amplitude and medium frequency pressures; (3) low amplitude and high frequency pressures caused by turbulence of the jet. It is highlighted in this paper that the jet bulge is responsible for the second part of the pressure oscillation.

\section{Experimental devices}

Figure 3 shows the experimental setup, which mainly consists of an air compressor 1 (maximum air pressure 3.0 MPa), a gas tank $2\left(0.5 \mathrm{~m}^{3}\right.$ volume $)$, a pressure adjusting valve 3 , a solenoid valve 4 , a nozzle assembly 5 and a water tank 6 ( $150 \mathrm{~cm}$ long, $5 \mathrm{~cm}$ wide and $110 \mathrm{~cm}$ high). A digital highspeed camera 7 (FASTCAM-super10KC, PHOTRON Co. Ltd., Japan) is used to visualize the flow field. The image signals are sent to a computer 11 through the controller 9 . A back light 8 is applied as the light source. 
Fig. 3 Schematic view of the experimental system. 1 , Air compressor; 2, gas tank; 3 , pressure adjusting valve; 4 , solenoid valve; 5 , nozzle assembly; 6, water tank; 7 , high-speed camera; 8 , light source; 9 , controller; 10, monitor; 11, computer

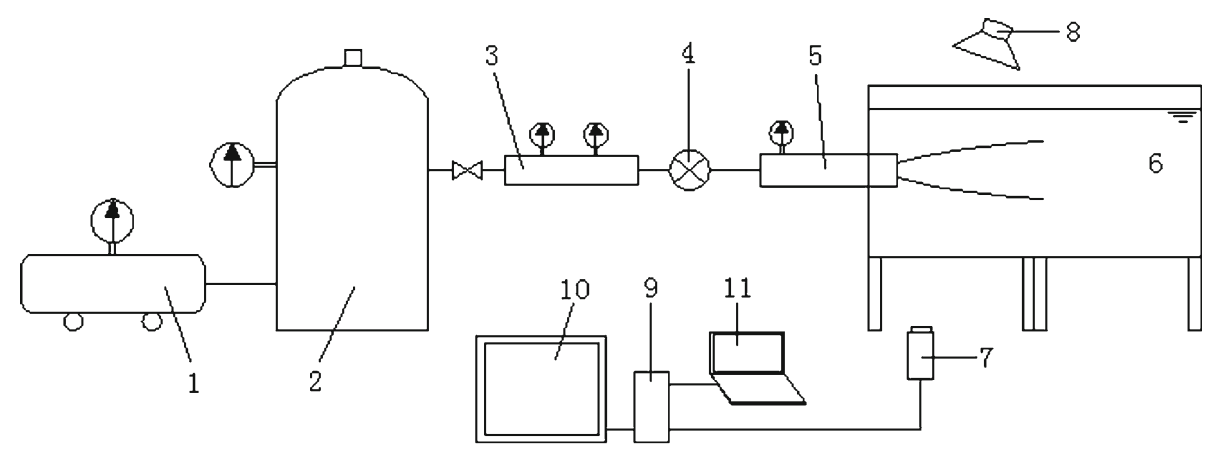

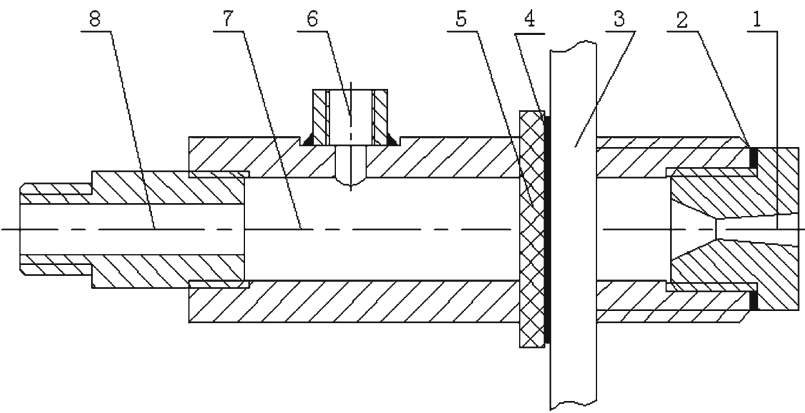

Fig. 4 Nozzle assembly. 1, Nozzle; 2, sealing ring; 3, side wall of the water tank; 4 , sealing ring; 5 , fastening ring; 6 , pressure gauge connector; 7 , gas chamber; 8 , connector of solenoid valve

The nozzle assembly is shown in Fig. 4. It is fastened on the wall of the water tank 3 by the fastening ring 5 . The nozzle assembly is aligned in horizontal position. In this paper's experiment, the nozzle throat and exit diameters are 4.0 and $6.5 \mathrm{~mm}$, respectively, and its design Mach number (full-expansion condition) is $\mathrm{Ma}=2.5$. In each experiment, the water depth from the free surface to the nozzle center line is $30 \mathrm{~cm}$ and the water is at room temperature. The environmental pressure at the nozzle exit $p_{b}$ is close to the atmospheric value. By changing the gas pressure $p_{0}$ in the chamber 7 , jets with different velocities can be obtained. In accordance with aerodynamic theory [23], the design Mach number $\mathrm{Ma}=2.5$ is achieved when the pressure ratio $p_{0} / p_{b}$ ensures a full-expansion isentropic flow. When the pressure ratio $p_{0} / p_{b}$ is less or greater than the design value, we obtain over-expansion or under-expansion condition. In this paper, we show the results of over-expanded air jets. If as a first approximation it is assumed that the pressure behind the incident inclined shock wave becomes atmospheric, the jet Mach number Me as shown in Fig. 2 can be calculated from existing aerodynamic data of air [23].

\section{Results}

Figures 5 and 6 show the bulge and the expansion feedback phenomena of an underwater supersonic air jet $(\mathrm{Me}=$
1.304), respectively. As indicated in Fig. 5b, the jet flow along the downstream distance can generally be divided into three regimes: (1) momentum jet at the nozzle exit region; (2) buoyant jet at the middle section of the jet; (3) plume at the end section of the jet. In the first regime, the jet shape remains straight or is slightly diverging due to the high velocity of the jet but the jet diverging angle is not as significant as suggested by Ozawa and Mori [10]. After the jet penetrates into the liquid, buoyancy makes the jet bend towards the free surface and the buoyant jet eventually becomes a plume.

The most important feature in Fig. 5 is that in the region of momentum jet, the jet bulge occurs as marked as I in Fig. 5c, f, h and k. Although the bulges exist only briefly $(\sim 1 \mathrm{~ms})$ and are swept downstream by the flow, their appearance means that the shock waves system in the jet is re-structured and the waves' energy is accumulated in a new way. This directly results in the consequence of the jet expansion feedback (see mark II in Fig. 6d). When the expansion feedback occurs, the jet reversely blows on the nozzle surface (Fig. 6b, c). Meanwhile, the jet suddenly expands radially and its diameter in Fig. 6e-h has been close to the nozzle outer diameter. Wang et al. [18] have shown that besides the radial expansion, the jet also expands downstream. From 2,000 pictures of the high-speed photographs, the frequencies of the bulge and the wave feedback are calculated to be $f_{b}=55.57 \mathrm{~Hz}$ and $f_{w}=8.55 \mathrm{~Hz}$, respectively.

As seen in Fig. 7, the length of the momentum jet region has been increased because of the increase of the jet velocity. Jet bulging (see mark I in Fig. 7d, g, k) and jet expansion feedback (see mark II in Fig. 8d) are also found. It is interesting to note that before the feedback occurs, the jet contraction (Fig. 8a) and flapping (Fig. 8b) appear. The jet expansion in the feedback process has made its diameter be greater than the nozzle outer diameter (Fig. 8f-h). From 2000 pictures of the high-speed photographs, the frequencies of the bulge and the expansion feedback are calculated to be $f_{b}=$ $97.35 \mathrm{~Hz}$ and $f_{w}=5.00 \mathrm{~Hz}$, respectively. We can now understand that the medium amplitude and medium frequency pressures in Fig. 2 are caused by jet bulging. The jet bulging 

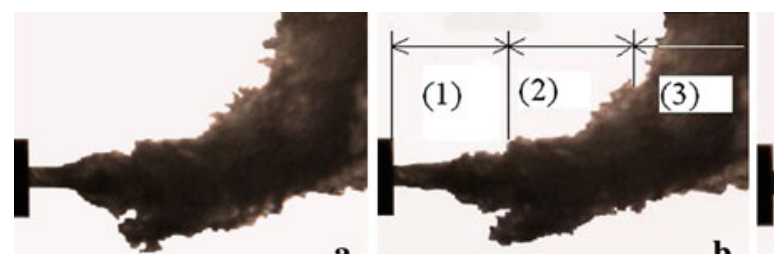

b
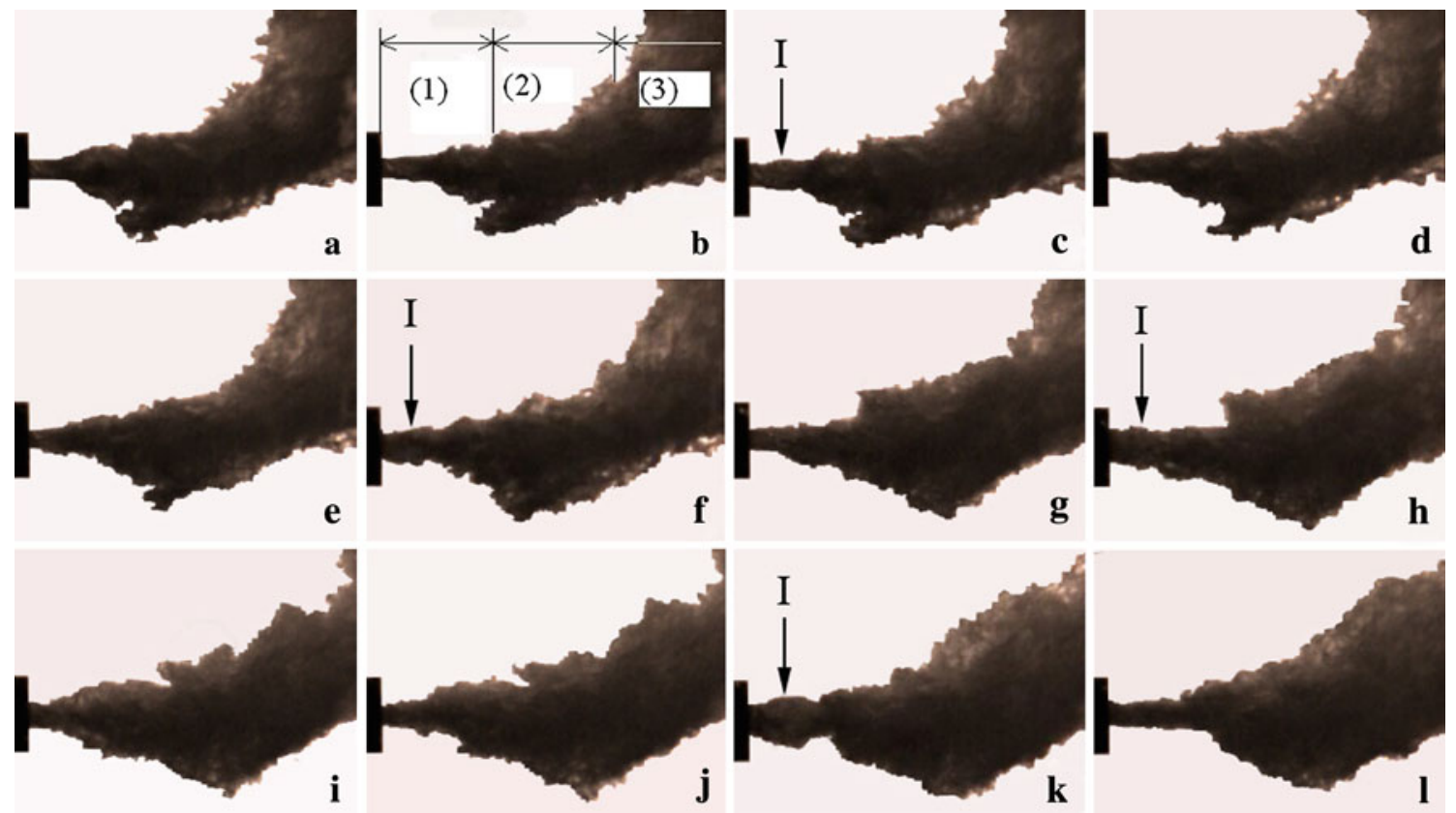

Fig. 5 High-speed photography of the bulge of an underwater overexpanded air jet. Interframe time $=1 \mathrm{~ms} . p_{0}=0.4 \mathrm{MPa}$. The jet Mach number at the nozzle exit is $\mathrm{Me}=1.304$. The water depth is $H=30 \mathrm{~cm}$.

The nozzle throat and exit diameters are 4.0 and $6.5 \mathrm{~mm}$. The nozzle outer diameter is $31 \mathrm{~mm}$. The bulge is marked as $I$ in the picture. Regime (1): momentum jet; Regime (2): buoyant jet; Regime (3): plume
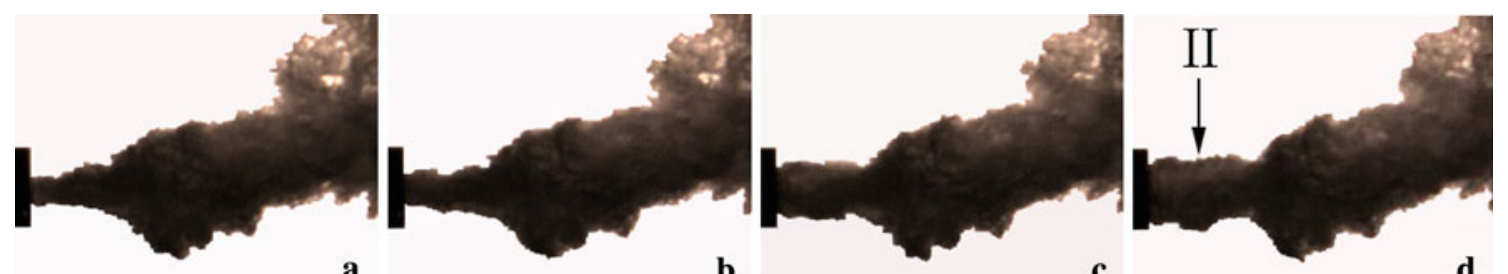
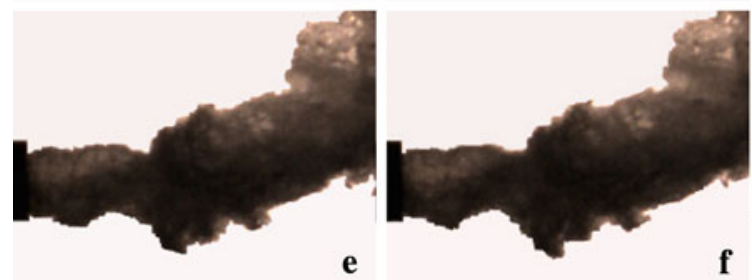

Fig. 6 High-speed photography of the jet expansion feedback of an underwater over-expanded air jet. Interframe time $=1 \mathrm{~ms} . p_{0}=$ $0.4 \mathrm{MPa}$. The jet Mach number at the nozzle exit is $\mathrm{Me}=1.304$. The

is the precursor of the reverse jet blowing off, namely, the jet expansion feedback.

In order to better understand the jet oscillation process, the variations of jet radius with time have been plotted in Fig. 9. Figures 5, 6, 7 and 8 are synchronized to Fig. 9. In the case of $p_{0}=0.4 \mathrm{MPa}$ (circular symbols in the figure), the jet diameter increases from 10 to $37 \mathrm{~mm}$ (maximum value) in less than $10 \mathrm{~ms}$ time. In the case of $p_{0}=$ $0.8 \mathrm{MPa}$ (triangle symbols in the figure), the maximum jet diameter of $40 \mathrm{~mm}$ appears at the time of $50 \mathrm{~ms}$. The maximum diameters are caused by the jet expansion feedback

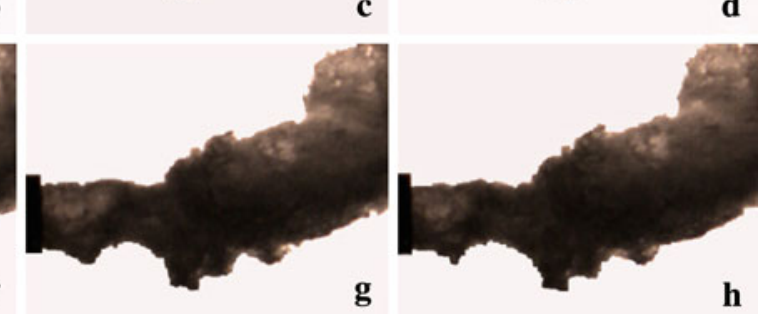

water depth is $H=30 \mathrm{~cm}$. The nozzle throat and exit diameters are 4.0 and $6.5 \mathrm{~mm}$. The nozzle outer diameter is $31 \mathrm{~mm}$. The feedback is marked as $I I$ in the picture

(back-attack). Before and after the maximum values of the radius, there are many times jet radius oscillations which have smaller amplitudes. These oscillations of jet radius are caused by the jet bulging.

\section{Conclusions}

The study of underwater supersonic gas jet covers gas-liquid two-phase flow physics and gasdynamics. This is why this topic is difficult. The current understanding to the mechanics 

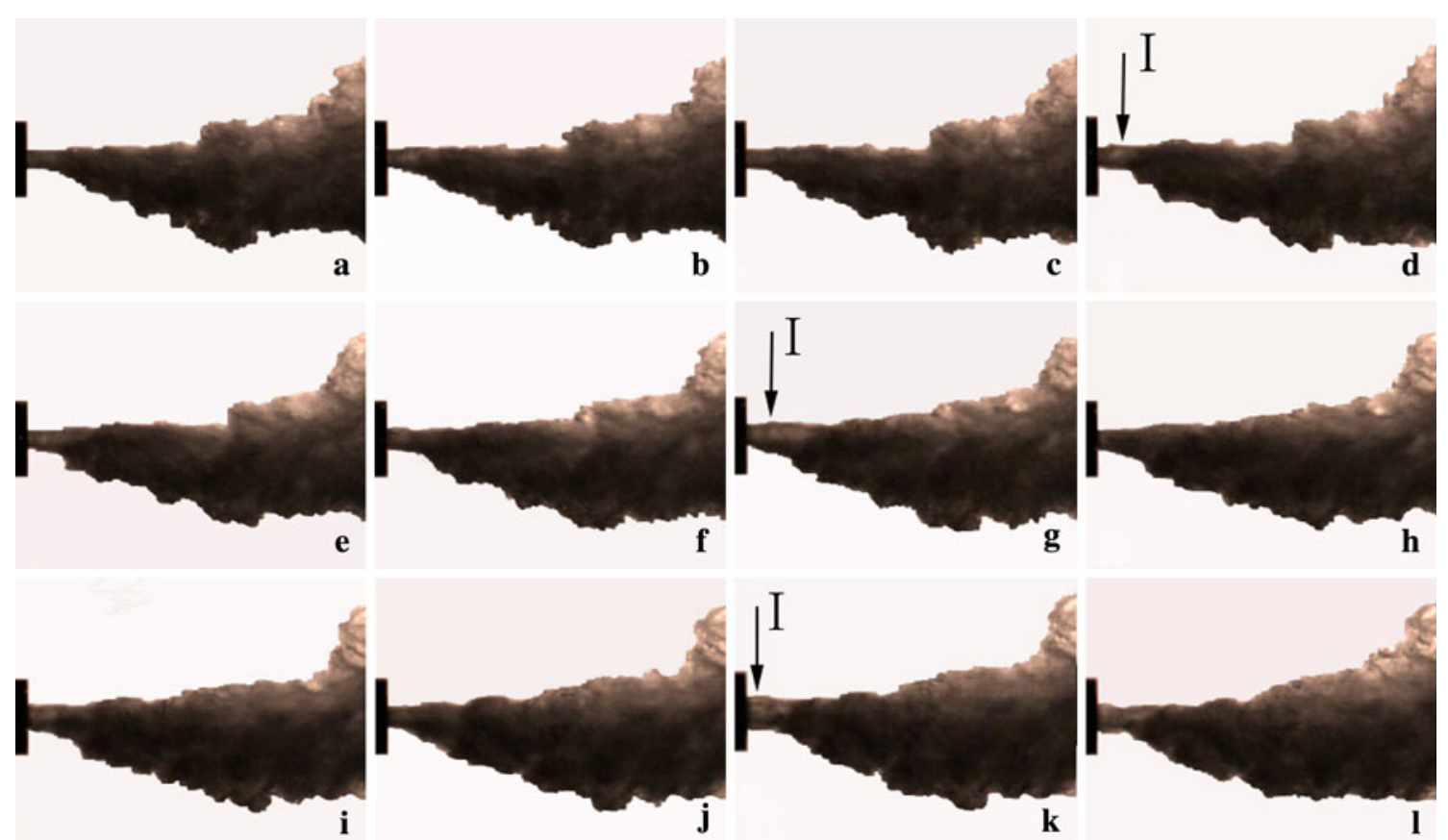

Fig. 7 High-speed photography of the bulge of an underwater over-expanded air jet. Interframe time $=1 \mathrm{~ms} . p_{0}=0.8 \mathrm{MPa}$. The jet Mach number at the nozzle exit is $\mathrm{Me}=1.954$. The water depth is
$H=30 \mathrm{~cm}$. The nozzle throat and exit diameters are 4.0 and $6.5 \mathrm{~mm}$. The nozzle outer diameter is $31 \mathrm{~mm}$. The bulge is marked as $I$ in the picture

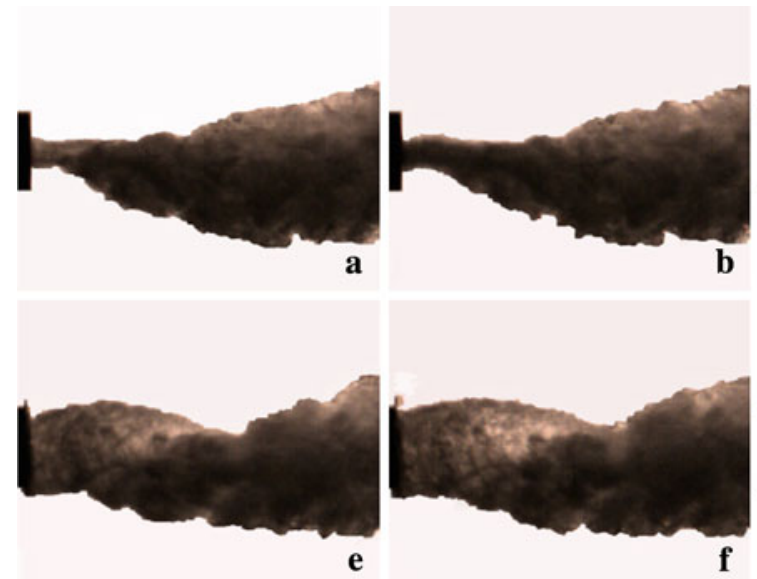

Fig. 8 High-speed photography of the jet expansion feedback of an underwater over-expanded air jet. Interframe time $=1 \mathrm{~ms} . p_{0}=$ $0.8 \mathrm{MPa}$. The jet Mach number at the nozzle exit is $\mathrm{Me}=1.954$.

of the jet is not sufficient yet. Perhaps the most comprehensive research in the past was given by Aoki et al. [11] and Aoki [12], who found the "back-attack" phenomenon and explained that the "back-attack" is caused by the jet contraction. However, the flow field is far more complicated than that obtained by Aoki et al. [11] and Aoki [12]. The shock waves at the nozzle exit interact with the gas/liquid interface will bring about the Richtmyer-Meshkov instability [24]. The Kelvin-Helmholtz instability at the shearing
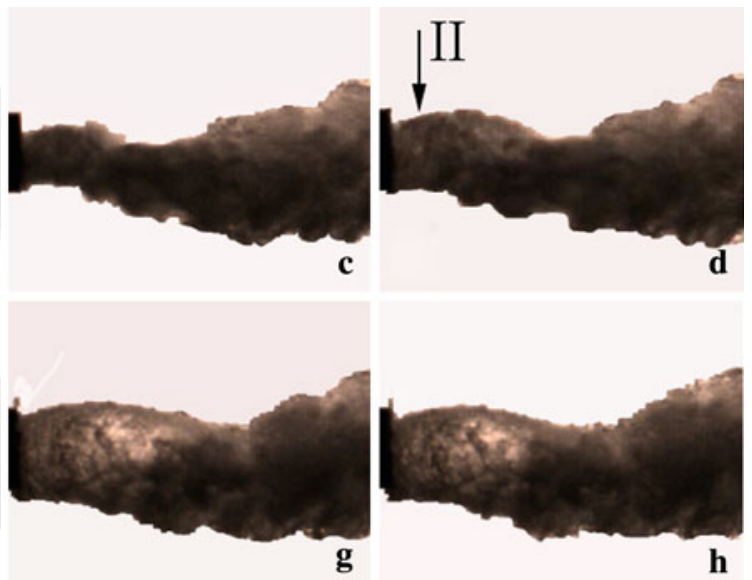

The water depth is $H=30 \mathrm{~cm}$. The nozzle throat and exit diameters are 4.0 and $6.5 \mathrm{~mm}$. The nozzle outer diameter is $31 \mathrm{~mm}$. The feedback is marked as $I I$ in the picture

layer may also play a role. There are other factors which may influence the jet boundary, such as buoyancy and turbulence. Therefore, the shock waves in the jet have to re-group and accumulate their energy again. This paper's experiment has demonstrated that for underwater supersonic gas jets, the bulge and the expansion feedback all appear. The frequency of the jet bulging is several times of the frequency of the expansion feedback. The jet diameter expands quickly in the wave feedback process and can be about 5 times of the 


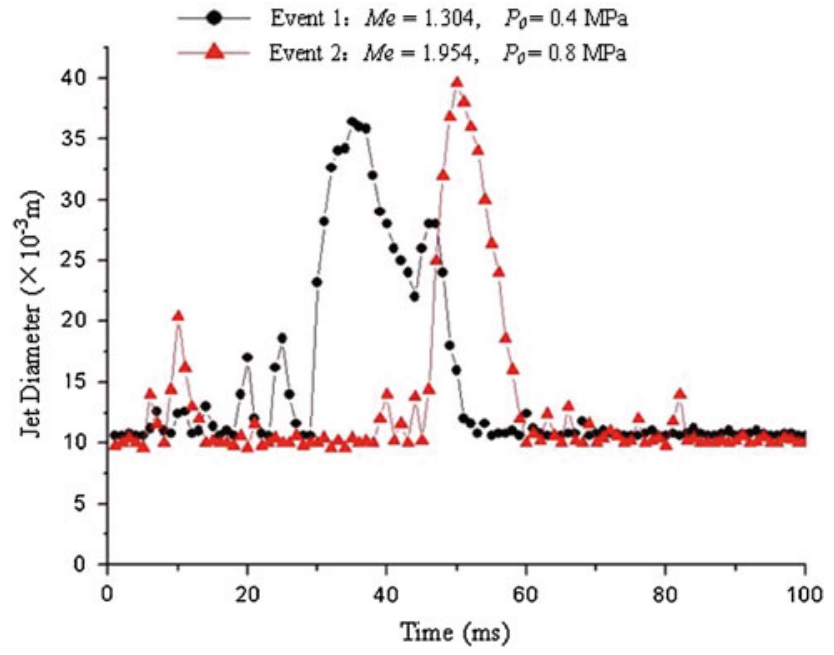

Fig. 9 Variation of jet radius with time before and after an expansion feedback. The location is at $10 \mathrm{~mm}$ downstream from the nozzle exit. Symbol $\bullet$ represents Event 1, $p_{0}=0.4 \mathrm{MPa}, \mathrm{Me}=1.304$; Symbol $\boldsymbol{\Delta}$ represents Event 2, $p_{0}=0.8 \mathrm{MPa}, \mathrm{Me}=1.954$

original diameter. The jet bulging is a short time process $(\sim 1 \mathrm{~ms})$. The appearance of the jet reverse impact on the nozzle surface is also a short time process $(\sim 1 \mathrm{~ms})$ although the jet expansion can last longer than $10 \mathrm{~ms}$. These all suggest that the jet bulging and the jet reverse blowing are kinds of shock wave phenomena. Our experiments at different conditions, which include the use of different kinds of nozzles, give identical conclusions.

Acknowledgments This study was supported by the National Natural Science Foundation of China (Grant no. 19972069 and 10672144), the Natural Science Foundation of Zhejiang Province of China (Grant no. Y107073.) and the Science Foundation of Zhejiang Sci-Tech University. The reviewers are thanked for their comments.

\section{References}

1. Meyer, R.X.: Hydrodynamics of the water and gas jet that initiated the Lake Nyos catastrophe. J. Fluids Eng. 126(5), 493-494 (2004)

2. Gulawani, S.S., Deshpande, S.S., Joshi, J.B., Shah, M.S., Prasad, C.S.R., Shukla, D.S.: Submerged gas jet into a liquid bath: a review. Ind. Eng. Chem. Res. 46, 3188-3218 (2007)

3. Ferguson, J.D.: Experimental data for underwater conical nozzles exhausting $\mathrm{N}_{2}$ gas. J. Hydronautics 3(4), 200 (1969)

4. Todman, M., Wallis, A.: Jet age steam power for marine propulsion. In: Ship Design and Operation for Environmental Sustainability. The Royal Institute of Naval Architects, London (2002)

5. Linck, M.B., Gupta, A.K., Bourhis, G., Yu, K.: Combustion characteristics of pressurized swirling spray flame and unsteady twophase exhaust jet. AIAA Paper 2006-377 (2006)
6. Weiland, C., Yagla, J., Vlachos P.: Experimental study of the stability of a high-speed gas jet under the influence of liquid crossflow. In: Proceedings of ASME/JSME 5th Joint Fluids Engineering Summer Meeting, vol.1, pp. 599-610, Paper No. FEDSM200737303, San Diego, California, USA, July 30-August 2 (2007)

7. Link, M., Gupta, A.K., Yu, K.: Submerged combustion and two-phase exhaust jet instabilities. J. Propuls. Power 25(2), 522532 (2009)

8. Hoefele, E.O., Brimacombe, J.K.: Flow regime in submerged gas injection. Metall. Trans. B 10, 631-648 (1979)

9. Ozawa, Y., Mori, K., Sana, M.: Behavior of injected gas observed at the exit of a submerged orifice in liquid metal. Tetsu-to-Hagane, 67(16), 2655-2664 (1981) (in Japanese)

10. Ozawa, Y., Mori, K.: Characteristics of Jetting observed in gas injection into liquid. Trans. ISIJ 23, 764-768 (1983)

11. Aoki, T., Masuda, S., Hatano, A., Taga, M.: Characteristics of submerged gas jets and a new type bottom blowing tuyere. In: Wraith, A.E. (ed.) Injection Phenomena in Extraction and Refining, pp. A136. University of Newcastle upon Tyne, Newcastle (1982)

12. Aoki, T.: The mechanism of the back-attack phenomenon on a bottom blowing tuyere investigated in model experiments. Tetsuto-Hagane, 76(11), 1996-2003 (1990) (in Japanese)

13. Shi, H.H., Wang, B.Y., Qi, L.X., Dai, Z.Q.: A submerged supersonic gas jet. In: Proceedings of 7th National Conference on Hydrodynamics, pp.75-81. Ocean Press, Beijing, (2005) (in Chinese)

14. Qi, L.X., Cao, Y., Wang, B.Y.: Experimental study of underexpanded sonic air jets in water. Acta Mechanica Sinica 32(6), 667-675 (2000) (in Chinese)

15. Loth, E., Faeth, G.M.: Structure of underexpanded round air jets submerged in water. Int. J. Multiph. Flow 15(4), 589-603 (1989)

16. Shao, S.F., Yan, J.J., Cao, Y., Liu, J.P.: Experimental research on flow and condensation of supersonic steam jet immerged in subcool water. J. Eng. Thermophys. 28(1), 68-70 (2007) (in Chinese)

17. Dai, Z.Q., Wang, B.Y., Qi, L.X., Shi, H.H.: Experimental study on hydrodynamic behavior of high-speed gas jet in still water. Acta Mech. Sinica 22, 443-448 (2006)

18. Wang, B.Y., Dai, Z.Q., Qi, L.X., Shi, H.H.: Experimental study on back-attack phenomenon in underwater supersonic jets. Chin. J. Theor. Appl. Mech. 39(2), 267-272 (2007) (in Chinese)

19. Lee, I.C., Lee, D.J.: An analysis of flow and screech tone from supersonic axisymmetric jet at the initial stage. J. Mech. Sci. Technol. 22, 819-826 (2008)

20. Tam, C.K.W., Viswanathan, K., Ahuja, K.K., Panda, J.: The source of jet noise: experimental evidence. J. Fluid Mech. 615, 253292 (2008)

21. Shi, H.H., Wang, B.Y., Dai, Z.Q.: Research on the mechanics of underwater supersonic gas jets. Sci China Phys. Mech. Astron. 53(3), 527-535 (2010)

22. Wang, X.G., Wang, C., Guo, Q., Shi, H.H.: Study on the vibrating flow type of submerged high-speed gas jets in a two-dimensional water tank. J. Zhejiang Sci-Tech Univ. 26(4), 613-618 (2009) (in Chinese)

23. Anderson, J.D. Jr.: Modern Compressible Flow. 2nd edn. McGrawHill, New York (1990)

24. Shi, H.H., Zhuo, Q.W.: Shock wave induced instability at a rectangular gas/liquid interface. In: Proceedings of 26th International Symposium on Shock Waves, vol. 2, pp. 1211-1216. Göttingen, Germany, July 15-20 (2007) 